LA-UR-98-4571

Aporioved for public release: cistribution is unlimited.

Title:

Magnetized Target Fusion (MTF) :

A Low-Cost Fusion Development Path

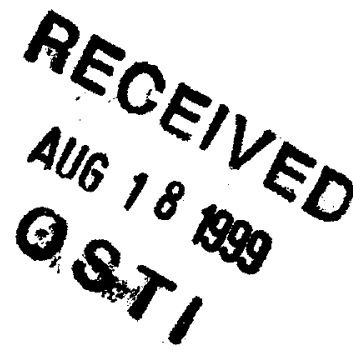

Author(s):

I. R. Lindmuth, R. E. Siemon, R. C. Kirkpatrick, .

R. E. Reinovsky

Submitted to:

Megagauss Magnetic Field \& Related Topics

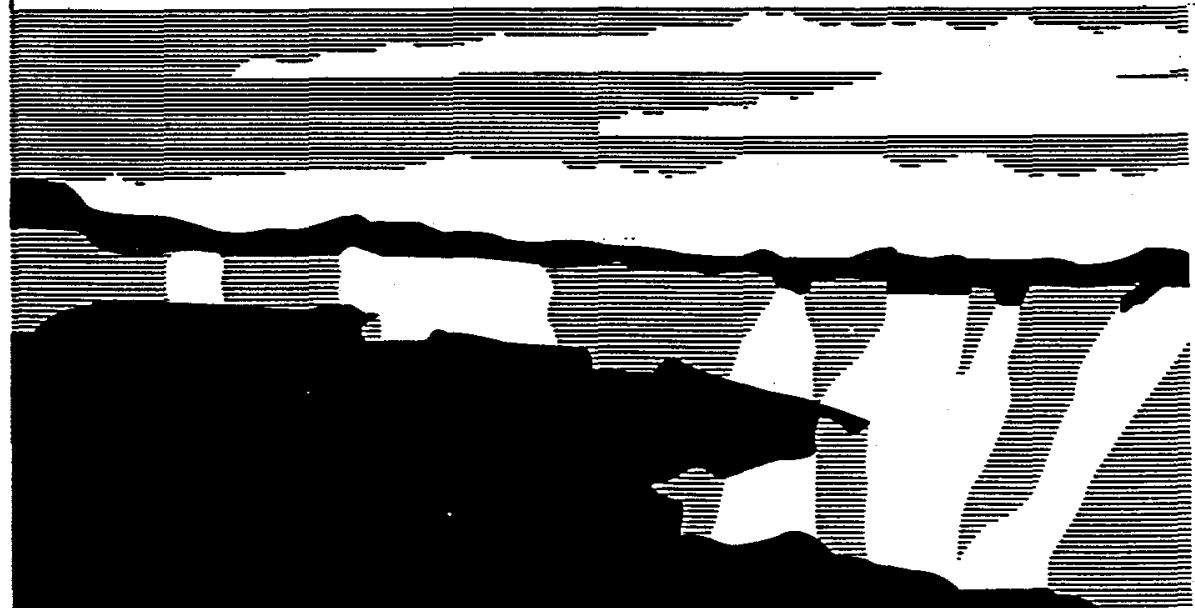

Los Alamos National Laboratory, an affimative action/equal opportunity employer, is operated by the University of Califormia for the U.S. Department of Energy under contract W-7405-ENG-36. By acceptance of this article, the pubilisher recognizes that the U.S. Govermment retains a nonexclusive, royalty-free license to putish or reproduce the putdished form of this contribution, or to allow others to do so, for U.S. Govemment purposes. Los Alamos National Laboratory requests that the publisher identify this article as work performed under the ausplces of the U.S. Department of Energy. The LOS Alamos National Laboratory strongly supports academic freedom and a researcher's right to publish; as an institution, however, the Laboratory does not endorse the viewpoint of a publication or guarantee its technical correctness. 


\section{DISCLAIMER}

This report was prepared as an account of work sponsored by an agency of the United States Government. Neither the United States Government nor any agency thereof, nor any of their employees, make any warranty, express or implied, or assumes any legal liability or responsibility for the accuracy, completeness, or usefulness of any information, apparatus, product, or process disclosed, or represents that its use would not infringe privately owned rights. Reference herein to any specific commercial product, process, or service by trade name, trademark, manufacturer, or otherwise does not necessarily constitute or imply its endorsement, recommendation, or favoring by the United States Government or any agency thereof. The views and opinions of authors expressed herein do not necessarily state or reflect those of the United States Government or any agency thereof. 


\section{DISCLAIMER}

Portions of this document may be illegible in electronic image products. Images are produced from the best available original document. 
LA-UR- $98-458$

LA-UR-98-

\title{
Magnetized Target Fusion (MTF): \\ A Low-Cost Fusion Development Path
}

\author{
Irvin R. Lindemuth, Richard E. Siemon, Ronald C. Kirkpatrick, \\ Robert E. Reinovsky \\ Los Alamos National Laboratory \\ Los Alamos NM USA
}

October 16, 1998

This is the text of an invited paper to be published in the Proceedings of the Eighth International Conference on Megagauss Magnetic Field Generation and Related Topics, Tallahassee, Florida, October 19-23, 1998.

Los Alamos National Laboratory is operated by the University of Califomia for the United States Department of Energy under contract W-7405-ENG-36.

By acceptance of this article, the publisher recognizes that the U.S. Government retains a nonexclusive, royalty-free license to publish or reproduce the published form of this contribution, $\alpha$ to allow others to do so, for US Government purposes.

Since changes may be made prior to publication, this preprint is made available with the understanding that it will not be cited without prior permission of the authors. 


\title{
Magnetized Target Fusion (MTF): A Low-Cost Fusion Development Path
}

\author{
Irvin R. Lindemuth, Richard E. Siemon, Ronald C. Kirkpatrick, \\ Robert E. Reinovsky \\ Los Alamos National Laboratory \\ Los Alamos NM USA
}

\begin{abstract}
Simple transport-based scaling laws are derived to show that a density and time regime intermediate between conventional magnetic confinement and conventional inertial confinement offers attractive reductions in system size and energy when compared to magnetic confinement and attractive reductions in heating power and intensity when compared to inertial confinement. This intermediate parameter space appears to be readily accessible by existing and near term pulsed power technologies. Hence, the technology of the Megagauss conferences opens up an attractive path to controlled thermonuclear fusion.
\end{abstract}

\section{Introduction}

In the more than three decades of the Megagauss conferences, remarkable progress has been made in magnetic flux compression technology for generating ultrahigh magnetic fields and intense, multimegampere electrical currents. One of the initial motivations for the development of this technology has been controlled thermonuclear fusion. However, it is only in light of recent developments that the technology of the Megagauss conferences can be considered to provide a complementary approach to the two conventional fusion approaches, magnetic confinement (MFE, or magnetic fusion energy), as now embodied primarily in tokamaks, and inertial confinement fusion (ICF), as now embodied primarily in laser driven targets. With proponents proposing $\alpha$ building two multi-billion dollar, multi-year next-generation facilities (ITER, or International Toroidal Experimental Reactor, for MFE, and NIF, or National Ignition Facility, for ICF), the conventional approaches have reached a funding crossroads. In this paper, we present arguments why controlled fusion may now be possible at substantially lower cost than the mainline approaches.

The plasma conditions that are required to achieve controlled thermonuclear fusion are well known. In general, these conditions are stated as an ion temperature, $T_{i}$, greater than $4 \mathrm{keV}$ and either a number-density/confinement-time product, $\mathrm{n} \tau$, greater than $10^{14} \mathrm{~s} / \mathrm{cm}^{3}$ (the "Lawson criterion" for MFE) or an areal density, $\rho R$, greater than $0.4 \mathrm{~g} / \mathrm{cm}^{2}$. It is the inability of any approach to obtain all of the required conditions simultaneously that has frustrated fusion researchers, and even after forty years of increasing knowledge about the behavior 
of plasmas, there remains no guarantee that next-generation machines, after a major capital investment, will finally achieve the long sought goal.

More than four docades of plasma research has shown that plasma "transport" presents the major impediment to achieving fusion conditions. As shown recently by Siemon $e t$ al. [1], the required energy and physical size of both MFE and ICF systems can be estimated with knowledge of the applicable diffusion coefficient that determines plasma transport. The beginning point for such estimates is a diffusion equation that is integrated over a volume and Gauss's theorem applied:

$$
\frac{\partial \mathrm{Q}}{\partial t}=-\nabla \cdot(D \nabla \mathrm{Q}) \Rightarrow \frac{\mathrm{QV}}{\tau}=-\mathrm{SD} \nabla \mathrm{Q}
$$

where $Q$ can be a plasma quantity such as density, temperature, or magnetic field, $D$ is the diffusion coefficient, $\tau$ is the characteristic transport time, $V$ is the volume, $S$ is the surface enclosing the volume, and, in the right-hand equation, $\mathrm{Q}$ and $\nabla \mathrm{Q}$ are "average" quantities. The average gradient, the volume, and the surface can be related to a characteristic dimension, $a$, as:

$$
\nabla \mathrm{Q}=-\frac{\mathrm{Q}}{\alpha a} ; \quad \mathrm{V}=\varepsilon a^{3} ; \quad \frac{\mathrm{V}}{\mathrm{S}}=\gamma a
$$

where $\alpha, \varepsilon$, and $\gamma$ are geometric factors.

We now make the observation that for minimum system size, the fusion burn time and the characteristic plasma heating time must be comparable to the transport characteristic time, i.e.,

$$
\tau \approx \tau_{\text {bum }} \approx \tau_{\text {heat }} ; \quad \tau_{\text {buen }}=\frac{L}{n}
$$

where $L$ is the Lawson criterion and $n$ is the plasma ion density. Combining Eqs. 1-3, we arrive at an estimate for the minimum fusion system size:

$$
a^{2}=\frac{D L}{\alpha m}
$$

Once the size is determined, the corresponding minimum plasma mass, minimum thermal energy, and minimum heating power, respectively, required for the fusion fuel follow:

$$
M=n m_{i} \varepsilon a^{3} ; \quad E_{p}=3 n k T \varepsilon a^{3} ; \quad P_{h}=\frac{E_{p} n}{L}
$$


where $m_{i}$ is the average ion mass, $k$ is the Boltzmann constant, and $T$ is the plasma temperature.

Eqs. 4 and 5 give minimum required plasma parameters in terms of the transport diffusion coefficient, the plasma temperature, the Lawson criterion, the plasma density, and geometry dependent quantities. Various plasma transport diffusion coefficients can be found in the literature. For example, Gross [2] gives ion and electron thermal conductivities, which can be related to a diffusion coefficient by the relationship $\mathrm{D}=K /(3 n k)$, where $K$ is the appropriate thermal conductivity. As shown by Gross, the thermal conductivity perpendicular to a magnetic field can be substantially rectuced when compared to the thermal conductivity without a magnetic field. This provides the basic motivation for magnetic confinement. However, as Gross and other authors discuss, many early attempts to confine plasmas magnetically encountered Bohm diffusion, a process that decreased inversely with the magnetic field, rather than inversely with the square of the magnetic field as the "classical" diffusion perpendicular to a magnetic field.

\section{Conventional approaches to fusion}

From Gross, we derive the following expressions for three plasma diffusion coefficients:

$$
\begin{aligned}
& \mathrm{D}^{\circ}=5.99 \times 10^{10} \frac{T^{5 / 2}}{n \ln \Lambda} \\
& \mathrm{D}^{\mathrm{c}}=4.53 \times 10^{13} \frac{\beta \ln \Lambda}{T^{3 / 2}} \\
& \mathrm{D}^{\mathrm{B}}=6.48 \times 10^{9} \frac{\beta^{1 / 2} T^{1 / 2}}{n^{1 / 2}}
\end{aligned}
$$

where $D^{\circ}$ is the electron-dominated thermal diffusion in the absence of a magnetic field, $D^{c}$ is the ion-dominated "classical" thermal diffusion across a magnetic field, and $\mathrm{D}^{\mathbf{B}}$ represents Bohm behavior. In Eq. 6 , the diffusion coefficients have units of $\mathrm{cm}^{2} / \mathrm{s}$ for temperature in Kelvin degrees and plasma density in $1 / \mathrm{cm}^{3}$. In Eq. 6 , we have introduced dimensionless quantities, $\beta$ (the ratio of plasma pressure to magnetic pressure) and the "Coulomb logarithm."

We now explore the implications of Eq. 6. For simplicity, in all of our subsequent analysis, we will use a plasma temperature $\mathrm{T}=1.16 \times 10^{3}{ }^{\circ} \mathrm{K}$ (10 $\mathrm{keV}$, a Lawson criterion, $L$, of $3 \times 10^{14} \mathrm{~s} / \mathrm{cm}^{3}$, and a pressure ratio $\beta=1$. We will also ignore the slow variation in the Coulomb logarithm, taking its value to be a constant with value 10 . And we will assume that the temperature 
gradient scale factor $\alpha=1$. We note that each of the diffusion coefficients in Eq. 6 scales differently with plasma density, $n$, and we note that the classical diffusion across the magnetic field is independent of $n$ at fixed $\beta$.

First consider the case of steady state behavior without a magnetic field. At $T=10 \mathrm{keV}$, the plasma pressure is given by $p$ (in atm) $=3.2 \times 10^{-14} / \mathrm{n}$. Steady state operation requires a pressure in the $1-10 \mathrm{~atm}$ range, e.g., $\mathrm{n}=10^{14} / \mathrm{cm}^{3}$. Using spherical geometry ( $\varepsilon=4.189, \gamma=0.333$ ) and substituting $D^{\circ}$ from Eq. 6 into Eq. 4 , we find that the minimum required system size is $2.79 \times 10^{8} \mathrm{~cm}$, i.e., approximately $20 \%$ of the diameter of the earth. Furthermore, Eqs. 4 and 5 lead to a required plasma thermal energy of $4.39 \times 10^{25} \mathrm{~J}$, an amount exceeding the energy received by the earth from the sun in some 300 days. Hence, we conclude that a steady-state, unmagnetized fusion system is impossible on earth.

At $10^{14} / \mathrm{cm}^{3}$, the Bohm diffusion coefficient of Eq. 6 is substantially smaller than the unmagnetized case. However, even a Bohm-like reduction in thermal conduction does not lead to a practical steady-state fusion system. Eqs. 4 and 5 give a characteristic dimension of $7.9 \mathrm{~km}$ and a required thermal energy of $1 \mathrm{GJ}$.

For toroidal geometry (e.g., tokamaks) with a major radius 3.5 times larger than the minor radius ( $\varepsilon=69.1, \gamma=0.5), D^{\epsilon}$ of Eq. 6 leads to a "classical" steadystate magnetized plasma system size of approximately $46.7 \mathrm{~cm}$. However, extensive research on toroidal geometries have to date found scaling approximately 30 times larger than classical, i.e., a diffusion coefficient of $10^{4}$ $\mathrm{cm}^{2} / \mathrm{s}$. This value leads to a system size of $245 \mathrm{~cm}$, which is to be compared to the ITER design minor radius of $171 \mathrm{~cm}$. According to Eq. 5 , the required thermal energy is $487 \mathrm{MJ}$ and the required heating power is $163 \mathrm{MW}$, numbers which are again comparable to ITER. It is the large volume and large heating requirements that lead to ITER's multi-billion dollar construction costs.

Whereas a steady-state, unmagnetized fusion system is impossible, ICF is based upon a pulsed system. According to Lindl [3], to match the NIF laser with target requirements, the target "hot spot" density will be about $3 \times 10^{25} / \mathrm{cm}^{3}$ (based upon an initial target radius of $0.087 \mathrm{~cm}$, a radial convergence of 36 , and a final areal density, $\rho R$, of about $0.3 \mathrm{~g} / \mathrm{cm}^{3}$ ). Note that this density is more than 11 crders of magnitude higher than the MFE steady-state density. For this density, the estimates above lead to a characteristic size of $9.4 \mu \mathrm{m}$ (compared to $24 \mu \mathrm{m}$ for NIF) and a heating power of $50 \mathrm{TW}$ (compared to the peak NIF laser power of $500 \mathrm{TW}$ ).

The plasma heating mechanism in ICF is compressional pdV heating. Eqs. 4 and 5 permit an estimate of the required implosion velocity. The heating intensity is the heating power, $P_{k}$, divided by the surface area, $S$. The corresponding implosion velocity is given by dividing the intensity by the plasma pressure:

$$
\mathrm{v}_{i}=\frac{P_{h} / S}{2 n k T}=\frac{3 \gamma}{2 L} a n
$$


Since $D^{\circ}$ of Eq. 6 is inversely proportional to $n, a$ (Eq. 4) is inversely proportional to $n$. Hence, the implosion velocity, $v_{i}$, is independent of $n$. Evaluating Eq. 7, we find that the required implosion velocity for ICF is 46.6 $\mathrm{cm} / \mu \mathrm{s}$, which is comparable to the NIF target velocity of $41 \mathrm{~cm} / \mu \mathrm{s}$ [3]. It is this high velocity, with the corresponding high power and intensity requirements, that leads to the multi-billion dollar cost of NIF.

\section{Magnetized Target Fusion}

The simple estimates give accurate parameters for MFE, where the fusion fuel is magnetized, and for ICF, where the fuel is unmagnetized. The same procedures can be applied to plasmas having megagauss magnetic fields. At 10 MG and $\beta=1$, the plasma density is $1.24 \times 10^{20} / \mathrm{cm}^{3}$, a value more-or-less the geometric mean of the densities for MFE and ICF. At this density, the bum time, for $L=3 \times 10^{14} \mathrm{~s} / \mathrm{cm}^{3}$, is $2.4 \mu \mathrm{s}$.

For $1.24 \times 10^{20} / \mathrm{cm}^{3}, \mathrm{D}^{\mathrm{c}}$ of Eq. 6 (classical transport) applied to cylinders with a length-to-diameter ratio of $3.3(\varepsilon=20.73, \gamma=0.5)$ leads to minimum required system size, mass, thermal energy, and heating power of $0.042 \mathrm{~cm}, 0.8$ $\mu \mathrm{g}, 3.37 \mathrm{~kJ}$, and $1.13 \mathrm{GW}$, respectively. The more pessimistic Bohm coefficient, $D^{B}$ of Eq. 6 , leads to $0.17 \mathrm{~cm}, 57 \mu \mathrm{g}, 65 \mathrm{~kJ}$, and $27 \mathrm{GW}$. In this intermediate density regime, the system sizes and energies are substantially lower than required for MFE while at the same time the required power is more than three orders of magnitude less than required for ICF.

To this point, our analysis of the intermediate density regime has been general and has not addressed a specific heating mechanism. However, if compressional heating is considered, then Eq. 7 gives an implosion velocity of $0.013 \mathrm{~cm} / \mu \mathrm{s}$ for classical diffusion and $0.05 \mathrm{~cm} / \mu \mathrm{s}$ for Bohm diffusion. Obviously, all estimates are minimum requirements and experience would suggest that minimum parameters should be substantially exceeded, if possible.

As evidenced by a number of papers in these proceedings, the time-scale, energy, and implosion velocity required at the intermediate density have already been exceeded by imploding liners. Hence, accessing the intermediate density regime by compressing a magnetized plasma by a magnetically driven liner appears particularly attractive. We refer to this approach as Magnetized Target Fusion (MTF), with the word "target" implying that the fuel is heated by implosion in a manner similar to that of ICF. In Russia, this approach is known as MAGO (see papers in these proceedings).

The simple estimates presented in this paper provide the motivation for MTF. Similar results using other plasma transport models have previously been reported [1]. More complete, fully time-dependent numerical simulations of magnetized targets can be found in the literature [e.g., 4,5]. These more complete models lead to the same conclusion: operating in the intermediate density regime has many potential advantages over the more conventional approaches. In fact, the parameter space in which magnetized targets are apt to 
work is far more extensive than the parameter space of the more conventional approaches.

The most obvious advantage of MTF is a potential cost reduction. As shown in this paper, the MFE constraint of steady-state operation and the ICF constraint of pulsed operation without magnetic fields lead to the requirements for expensive facilities. On the other hand, existing Disk Explosive Magnetic Generators (DEMG) and the Los Alamos Atlas capacitor bank aready under construction (see papers in these proceedings), and perhaps the Air Force Research Laboratory Shiva-Star capacitor bank, appear more than adequate to achieve the long-sought goal of "scientific break-even" in fusion research via MTF.

A number of researchers around the world have recognized the attractiveness of the intermediate density regime. In October, 1997, the First International Workshop on Magnetized Target Fusion was held in Pleasanton, $\mathrm{Ca}$. in conjunction with an IAEA Technical Committee Meeting on Innovative Approaches to Fusion Energy. At the combined meetings, MTF-related papers were presented by authors from the US, Russia, Canada, France, and New Zealand. A collection of presentations at these meetings is available from the authors of this paper. The presentations emphasized the point that various combinations of plasma formation systems and implosion systems appear feasible (see also papers in these proceedings).

We believe the technology of the Megagauss conferences opens up this attractive and unexplored path to controlled fusion. MTF is clearly still a research topic, and substantial fusion yield has not yet been demonstrated. Although some physics uncertainties exist, no insurmountable obstacles have been identified. The basic driver technology required for demonstration of MTF clearly exists. MTF is certainly an "orthogonal," complementary alternate to MFE and ICF. Because MTF is qualitatively different from MFE and ICF-different time, length, and density scales-MTF fusion reactors will have different characteristics and trade-offs, increasing the chances that a practical fusion power scheme can be found.

[1] "Why Magnetized Target Fusion Offers a Low-Cost Development Path for Fusion Energy," R. Siemon, I. Lindemuth, K. Schoenberg, accepted for publication in Comments on Plasma Physics and Controlled Fusion (1998).

[2] Fusion Energy, R. A. Gross.

[3] "Development of the Indirect-Drive Approach to Inertial Confinement Fusion and the target Physics Basis for Ignition and Gain," J. Lindl, Lawrence Livermore National Laboratory report UCRL-JC-119015 (1995).

[4] "Parameter Space for Magnetized Fuel Targets in Inertial Confinement Fusion," I. Lindemuth, R. Kirkpatrick, Nuclear Fusion 23, 263 (1983).

[5] "Ignition and Bum in Inertially Confined Magnetized Fuel," R. Kirkpatrick and I. Lindemuth, Fusion Technology 20, 834 (1991). 